NASA/TM-2014-218305

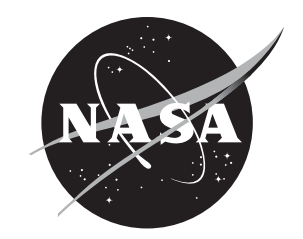

\title{
Magnetic Tunnel Junction-Based On-Chip Microwave Phase and Spectrum Analyzer
}

Xin Fan, Yunpeng Chen, Yunsong Xie, and James Kolodzey

University of Delaware, Newark, Delaware

Jeffrey D. Wilson and Rainee N. Simons

Glenn Research Center, Cleveland, Ohio

John Q. Xiao

University of Delaware, Newark, Delaware 


\section{NASA STI Program . . . in Profile}

Since its founding, NASA has been dedicated to the advancement of aeronautics and space science. The NASA Scientific and Technical Information (STI) program plays a key part in helping NASA maintain this important role.

The NASA STI Program operates under the auspices of the Agency Chief Information Officer. It collects, organizes, provides for archiving, and disseminates NASA's STI. The NASA STI program provides access to the NASA Aeronautics and Space Database and its public interface, the NASA Technical Reports Server, thus providing one of the largest collections of aeronautical and space science STI in the world. Results are published in both non-NASA channels and by NASA in the NASA STI Report Series, which includes the following report types:

- TECHNICAL PUBLICATION. Reports of completed research or a major significant phase of research that present the results of NASA programs and include extensive data or theoretical analysis. Includes compilations of significant scientific and technical data and information deemed to be of continuing reference value. NASA counterpart of peer-reviewed formal professional papers but has less stringent limitations on manuscript length and extent of graphic presentations.

- TECHNICAL MEMORANDUM. Scientific and technical findings that are preliminary or of specialized interest, e.g., quick release reports, working papers, and bibliographies that contain minimal annotation. Does not contain extensive analysis.

- CONTRACTOR REPORT. Scientific and technical findings by NASA-sponsored contractors and grantees.
- CONFERENCE PUBLICATION. Collected papers from scientific and technical conferences, symposia, seminars, or other meetings sponsored or cosponsored by NASA.

- SPECIAL PUBLICATION. Scientific, technical, or historical information from NASA programs, projects, and missions, often concerned with subjects having substantial public interest.

- TECHNICAL TRANSLATION. Englishlanguage translations of foreign scientific and technical material pertinent to NASA's mission.

Specialized services also include creating custom thesauri, building customized databases, organizing and publishing research results.

For more information about the NASA STI program, see the following:

- Access the NASA STI program home page at http://www.sti.nasa.gov

- E-mail your question to help@sti.nasa.gov

- Fax your question to the NASA STI Information Desk at 443-757-5803

- Phone the NASA STI Information Desk at 443-757-5802

- Write to: STI Information Desk NASA Center for AeroSpace Information 7115 Standard Drive Hanover, MD 21076-1320 
NASA/TM-2014-218305

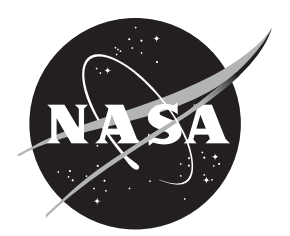

\section{Magnetic Tunnel Junction-Based On-Chip Microwave Phase and Spectrum Analyzer}

Xin Fan, Yunpeng Chen, Yunsong Xie, and James Kolodzey

University of Delaware, Newark, Delaware

Jeffrey D. Wilson and Rainee N. Simons

Glenn Research Center, Cleveland, Ohio

John Q. Xiao

University of Delaware, Newark, Delaware

National Aeronautics and

Space Administration

Glenn Research Center

Cleveland, Ohio 44135 


\section{Acknowledgments}

The University of Delaware authors acknowledge the support that they have received from the NASA EPSCoR Program under contract number NNX11AQ29A.

This report contains preliminary findings, subject to revision as analysis proceeds.

Level of Review: This material has been technically reviewed by technical management.

Available from

NASA Center for Aerospace Information 7115 Standard Drive

Hanover, MD 21076-1320
National Technical Information Service 5301 Shawnee Road Alexandria, VA 22312

Available electronically at http://www.sti.nasa.gov 


\title{
Magnetic Tunnel Junction-Based On-Chip Microwave Phase and Spectrum Analyzer
}

\author{
Xin Fan, Yunpeng Chen, Yunsong Xie, and James Kolodzey \\ University of Delaware \\ Newark, Delaware 19716 \\ Jeffrey D. Wilson and Rainee N. Simons \\ National Aeronautics and Space Administration \\ Glenn Research Center \\ Cleveland, Ohio 44135 \\ John Q. Xiao \\ University of Delaware \\ Newark, Delaware 19716
}

\begin{abstract}
A magnetic tunnel junction (MTJ)-based microwave detector is proposed and investigated. When the MTJ is excited by microwave magnetic fields, the relative angle between the free layer and pinned layer alternates, giving rise to an average resistance change. By measuring the average resistance change, the MTJ can be utilized as a microwave power sensor. Due to the nature of ferromagnetic resonance, the frequency of an incident microwave is directly determined. In addition, by integrating a mixer circuit, the MTJ-based microwave detector can also determine the relative phase between two microwave signals. Thus, the MTJbased microwave detector can be used as an on-chip microwave phase and spectrum analyzer.
\end{abstract}

\subsection{Introduction}

Spintronics explores phenomena intertwining electronic charge and spin (Ref. 1). The ability of spintronics to re-energize itself in directions that germinate new subfields has made it one of the most fertile grounds for basic research aimed at future applications. It has also crucially relied on the discovery of new materials and heterostructures where control over spin-dependent properties of interfaces is essential. The first-generation spintronics (Refs. 2 to 4) were primarily focused on giant magneto resistance (GMR) in FM (ferromagnetic)/NM (normal metal)/FM spin valves (SVs) and tunneling magneto resistance (TMR) in FM/I (insulator)/FM magnetic tunnel junctions (MTJs). The GMR and TMR are defined as $\left(R_{A P}-R_{P}\right) / R_{P}$, where $R_{A P}$ and $R_{P}$ are the resistances when magnetizations in two ferromagnetic layers are parallel and antiparallel, respectively. On the other hand, the so-called secondgeneration spintronics (Ref. 2) aim to harness spin coherence (precessing spins), in which a persistent component of the spin can be maintained transverse to an applied magnetic field or magnetization, as well as low-dissipation (i.e., not accompanied by any net charge flux) pure spin currents (Ref. 3).

The discovery of spintronic devices has already led to breakthroughs in hard disk recording technologies (Ref. 4). Intensive research is also underway to develop magnetic random access memory featuring non-volatility, high density, high speed, and low energy consumption (Ref. 4). The studies of second-generation spintronic devices have also promised an array of microwave devices where spin dynamics plays a key role. For example, a new type of microwave detector has been demonstrated in which the magnetization is precessed by microwaves in a nano- to micron-sized MTJ whose resistance change rectifies the input RF current, giving rise to a DC voltage that corresponds to the input microwave power (Ref. 5). Owing to the high TMR ratio, spin transfer torque, and voltage induced magnetic anisotropy, the $\mathrm{CoFeB} / \mathrm{MgO} / \mathrm{CoFeB}$-based MTJ microwave detector shows high sensitivity comparable to a conventional Schottky RF diode (Ref. 6). In addition, the microwave frequency can be determined by 
the nature of ferromagnetic resonance in MTJs, which removes the need of the mixer circuit in conventional RF diode detection. Besides the microwave magnitude and frequency, the phase detection has been demonstrated through using a single magnetic stripe (Ref. 7).

In this paper, we present our recent studies on the MTJ spin dynamics under microwave excitation and the development of an MTJ-based on-chip microwave spectrum analyzer. Section 2.0 establishes the basic principles of using an MTJ to detect microwave power and frequency. In Section 3.0, the potential application of the MTJ is extended to microwave phase detection and spectrum analysis. By combining MTJs in a simple mixing circuit, the interference of two microwave signals can be detected. Section 4.0 presents the conclusions.

\subsection{Microwave Power and Frequency Detector}

The resistance of an MTJ depends on the relative angle $\theta$ between the magnetizations in two ferromagnetic layers: $R=\frac{R_{A P}+R_{P}}{2}-\frac{R_{A P}-R_{P}}{2} \cos \theta$. One of the magnetic layers (pinned layer) is fixed by the under-layers and the other magnetic layer (free layer) is free to rotate in an external magnetic field (Ref. 8). Figure 1(a) shows a typical TMR curve of an MTJ with AlOx as the barrier layer. The MTJ has a 6 percent magneto resistance (MR) change under a DC bias of $10 \mu \mathrm{A}$. When the MTJ is exposed to microwave magnetic fields $\left(h_{r f}\right)$, the free layer magnetization precesses and the angle $\theta$ varies, resulting in a changing resistance. As shown in Figure 1(b), with an MTJ initially configured in a parallel configuration, the resistance of the MTJ has the lowest value (the red solid line). When the precession starts, the non-colinear magnetizations between the free layer and pinned layer always increases the resistance, giving rise to a higher average resistance (the yellow dashed line). On the other hand, if the MTJ is configured to be at an antiparallel configuration in Figure 1(c), the initial resistance of the MTJ is the highest. The magnetization precession results in a reduced average resistance. The change of the average resistance is related to the magnitude of the microwave magnetic field $h_{r f}$, and the magnetic susceptibility tensor $\chi$, which depends on the microwave frequency $f$, external bias magnetic field $H$ and the material properties of the MTJ.

When the microwave magnetic field intensity is lower than the spin wave instability threshold, the precession is linear to the applied microwave magnetic field.

The susceptibility tensor $\chi=\left[\begin{array}{ll}\chi_{x x} & \chi_{x y} \\ \chi_{y x} & \chi_{y y}\end{array}\right]$ in a thin magnetic film can be derived from the LaudauLifshitz-Gilbert equation (Ref. 9) as

$$
\left\{\begin{array}{l}
\chi_{x x}=\frac{\gamma M_{s}\left[\gamma\left(H+H_{a}+M_{s}\right)+i \alpha f\right]}{\left[\left(\gamma\left(H+H_{a}+M_{s}\right)+i \alpha f\right)\left(\gamma\left(H+H_{a}\right)+i \alpha f\right)-f^{2}\right]} \\
\chi_{x y}=-\chi_{y x}=\frac{i \gamma M_{s} f}{\left[\left(\gamma\left(H+H_{a}+M_{s}\right)+i \alpha f\right)\left(\gamma\left(H+H_{a}\right)+i \alpha f\right)-f^{2}\right]} \\
\chi_{y y=} \frac{\gamma M_{s}\left[\gamma\left(H+H_{a}\right)+i \alpha f\right]}{\left[\left(\gamma\left(H+H_{a}+M_{s}\right)+i \alpha f\right)\left(\gamma\left(H+H_{a}\right)+i \alpha f\right)-f^{2}\right]}
\end{array}\right.
$$

where $M_{s}$ is the saturation magnetization, $H$ is the external bias magnetic field, $\alpha$ is the damping constant, $\gamma$ is the gyromagnetic ratio, which is typically $28 \mathrm{GHz} /$ Tesla, $H_{a}$ is the effective anisotropy field, and $f$ is the frequency of microwave input. 

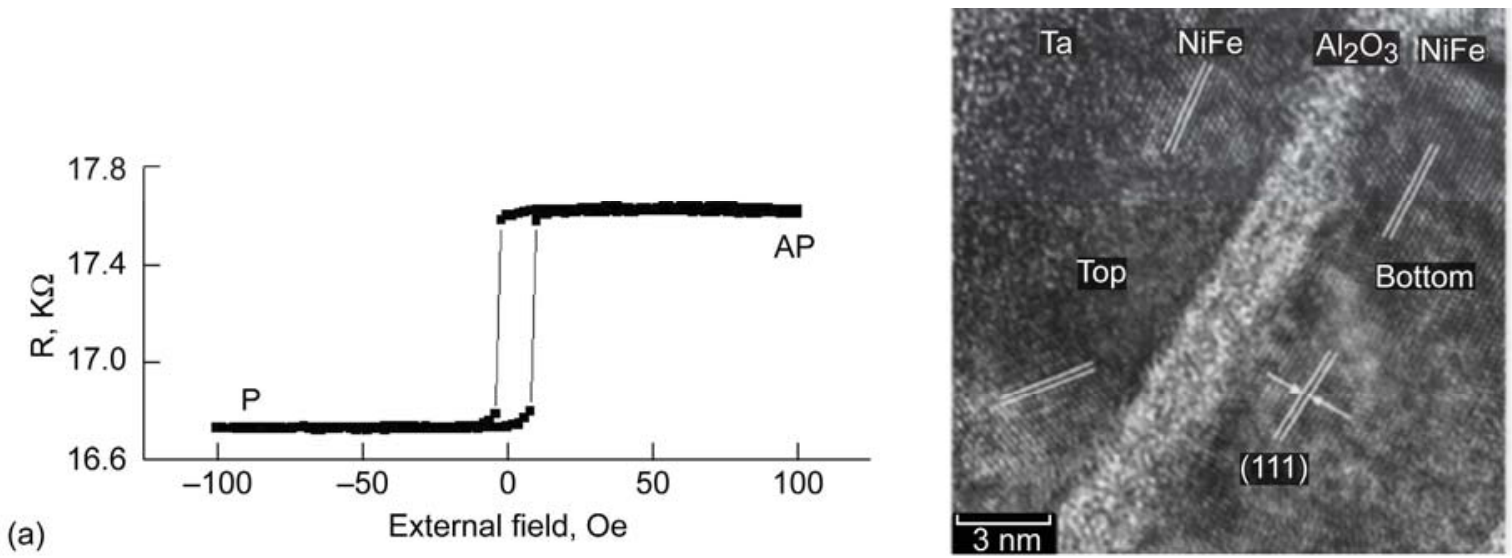

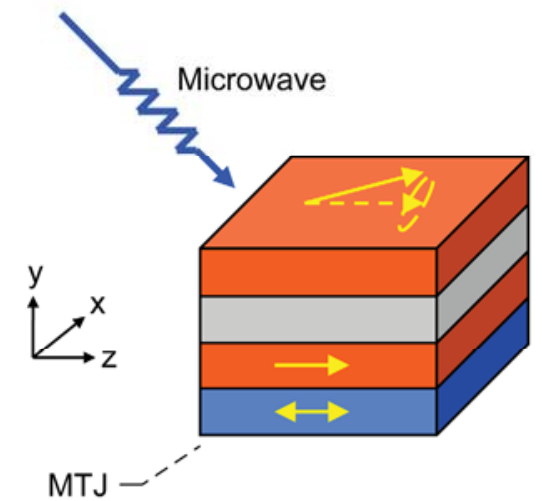

(b)

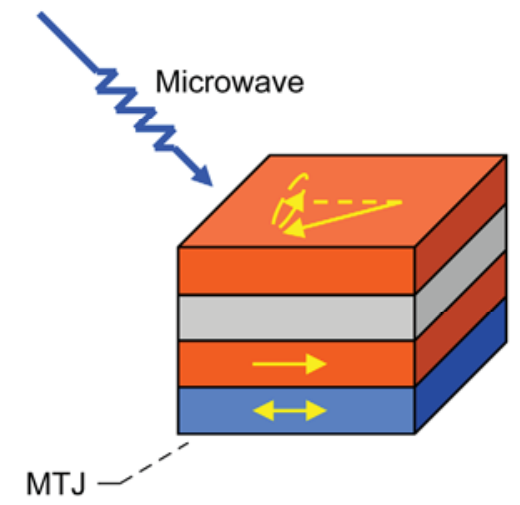

(c)

Figure 1.-(a) Typical TMR curves of MTJs and TMR image of the cross section of MTJs. (b) Illustration of magnetization precession and resistance change in MTJ under microwave excitation with parallel $(P)$ configuration. (c) Antiparallel (AP) configuration.
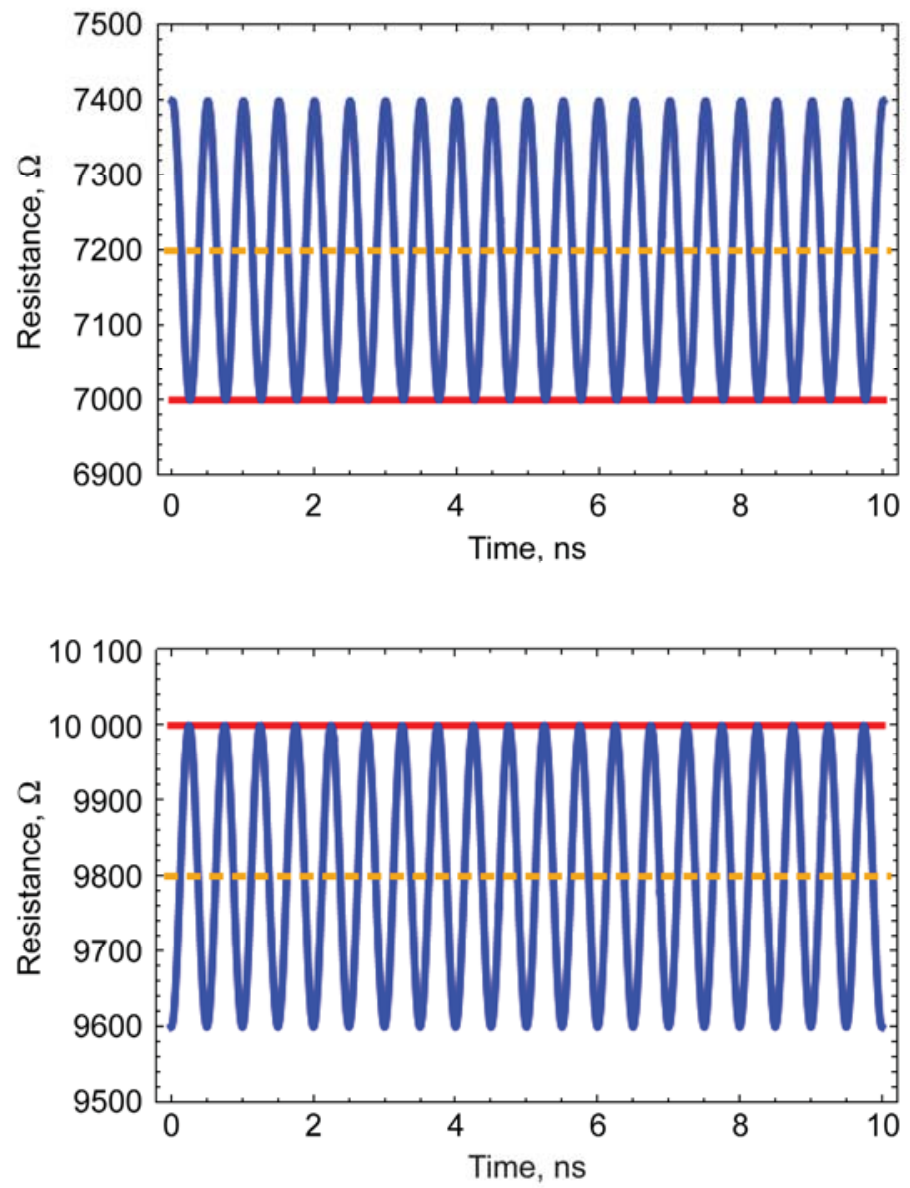
Because of the strong demagnetizing effect in a magnetic thin film, $\chi_{x x}>>\chi_{x y}>>\chi_{y y}$, the magnetization precession of the free layer excited by the microwave magnetic field is

$$
m_{\text {free }}(t)=\chi_{x x_{-} \text {free }} \cdot h_{r f}(t) .
$$

The average resistance change can then be expressed as

$$
\Delta R=-\frac{R_{A P}-R_{P}}{2}\langle\cos \theta(t)\rangle=\frac{R_{A P}-R_{P}}{8 M_{s}} h_{r f}^{2} \cos \theta_{0} \mid \chi_{x x_{-}} \text {free }\left.\right|^{2}
$$

where $\theta_{0}$ is the initial angle between the free and pinned layers. The microwave-induced resistance change is proportional to the square of magnetic susceptibility.

An experiment is carried out to demonstrate such resistance changes due to microwave irradiation. In order to detect the small resistance change, a lock-in technique is developed. As shown in Figure 2(a), a vector network analyzer (VNA) is used as a microwave source and an RF amplifier with nominal gain of $28 \mathrm{~dB}$ then amplifies the microwave signal. The microwave signal is fed into a coplanar waveguide $(\mathrm{CPW})$ with the center conductor width of about $50 \mu \mathrm{m}$. An MTJ is lithographically patterned on top of the CPW center line on a silicon wafer. A typical MTJ stack is $10 \mathrm{~nm} \mathrm{Cr} / 120 \mathrm{~nm} \mathrm{Cu} / 4 \mathrm{~nm} \mathrm{CoFe} / 15 \mathrm{~nm}$ $\mathrm{IrMn} / 4 \mathrm{~nm} \mathrm{CoFe} / 2.3 \mathrm{~nm} \mathrm{AlOx} / 20 \mathrm{~nm} \mathrm{NiFe}(\mathrm{Py}) / 20 \mathrm{~nm} \mathrm{Cu} / 50 \mathrm{~nm}$ Au. The bottom electrode containing $10 \mathrm{~nm} \mathrm{Cr} / 120 \mathrm{~nm} \mathrm{Cu}$ is used to pattern the CPW. When the microwave signal propagates through the $\mathrm{CPW}$, a microwave magnetic field is generated on top of the CPW center line with direction parallel to the x-axis. An electromagnet generates the DC bias magnetic field and the AC modulated field. The MTJ is biased by a constant current source and the voltage is monitored by a lock-in amplifier. Due to the modulation of the external magnetic field, the microwave-induced voltage can be expressed as

$$
\begin{aligned}
& \Delta V=I_{d c} \cdot \Delta R \propto|\chi[H+\Delta H(t)]|^{2}=|\chi[H]|^{2}+\frac{d|\chi[H]|^{2}}{d H} \Delta H(t)+ \\
& \frac{d^{2}|\chi[H]|^{2}}{2 d H^{2}} \Delta H(t)^{2}+\frac{d^{3}|\chi[H]|^{2}}{6 d H^{3}} \Delta H(t)^{3}+\frac{d^{4}|\chi[H]|^{2}}{24 d H^{4}} \Delta H(t)^{4} \\
& +O\left(\Delta H(t)^{5}\right)
\end{aligned}
$$

where $\Delta H(t)=\Delta H_{0} \cos \omega_{0} t$ is the AC modulation field. The first harmonic of the microwave-induced voltage is measured with a lock-in amplifier, as shown in Figure 2(b). The resonance frequency and field have a relation described by the Kittel equation, $f=\gamma \sqrt{H\left(H+M_{s}\right)}$. Therefore, the frequency of an unknown microwave signal can be determined by scanning the field. There is a large peak in the vicinity of magnetization switching and there is an overall drifting of the curve from a positive field to a negative field. This is due to the magnetization switching of the free layer and the misalignment of the pinned layer. A background signal may be used for calibration when no microwave signal is present. As shown in Figure 2(c), the value of the second order harmonic term is of a similar order of magnitude as that of the first order term while having less background signal and narrower line width. In real applications, the use of the second order harmonic term may be more practical than the use of the first order term. It should also be pointed out that the magnitude of field modulation is only 1.5 Oe in the experiment. Practically, a much higher voltage signal can be obtained when the magnitude of field modulation is close to the ferromagnetic resonance (FMR) line width of the MTJ. According to Equation (3), the microwaveinduced voltage is proportional to $h_{r f}^{2}$ and is thus linearly proportional to the microwave power. The relation between the microwave-induced voltage and the nominal input microwave power is shown in Figure 2(d). By using CPWs of small signal line width, we can significantly enhance the detection dynamic range by cascading CPWs of different sizes, each containing an MTJ detector. 
(a)

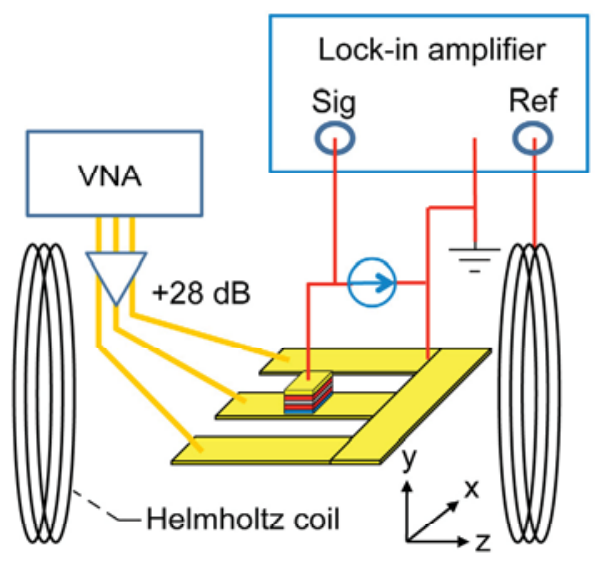

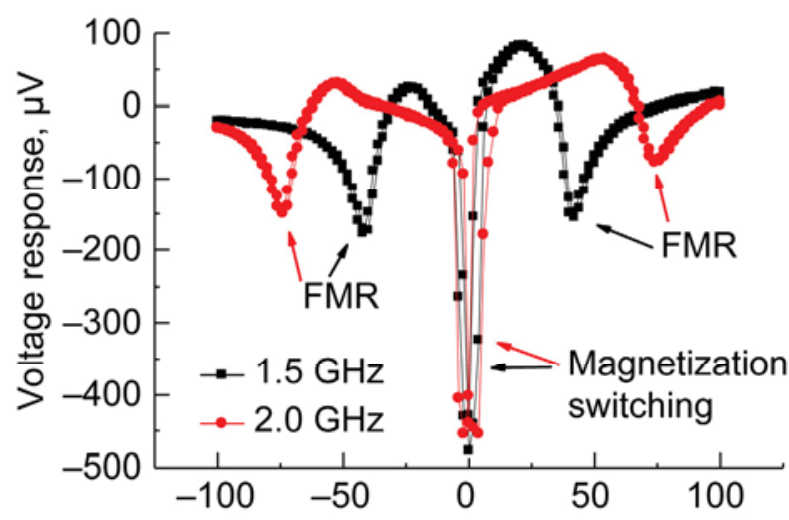

(b)

External field, Oe

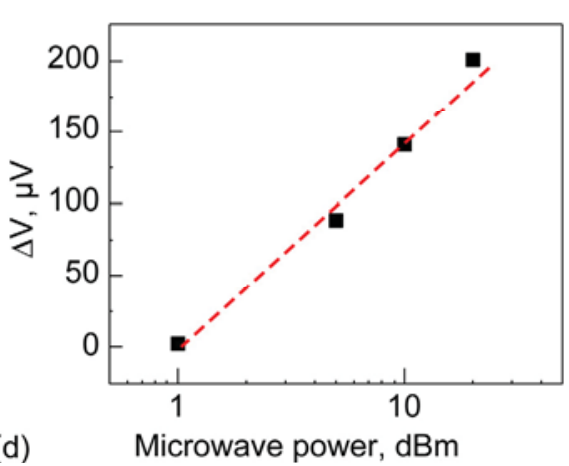

(d)

(c)
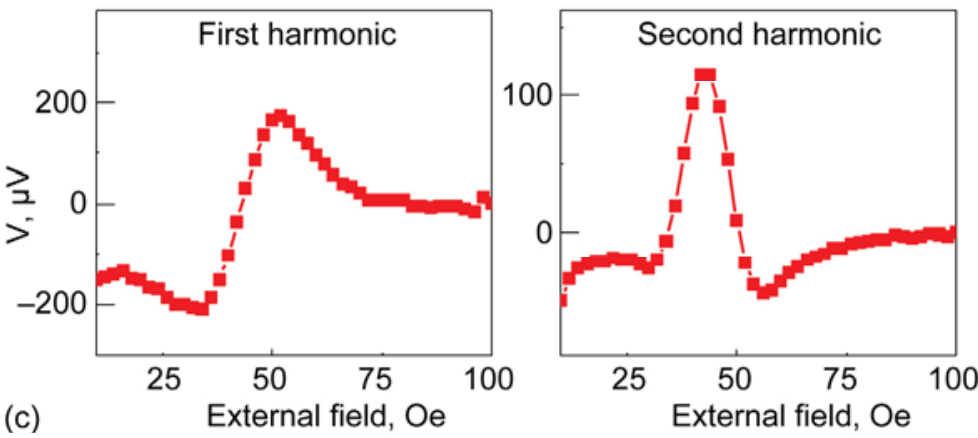

Figure 2.-(a) Field-modulated microwave detection. (b) Example of a microwave-induced voltage signal curve. The nominal microwave power is $20 \mathrm{dBm}$, and the nominal modulation field is $1.5 \mathrm{Oe}$ with frequency of $73 \mathrm{~Hz}$. A strong peak is observed in the vicinity of magnetization reversal. (c) The first and second harmonic signals at $2 \mathrm{GHz}$. (d) Power dependence of microwave-induced 1st harmonic signal at $2 \mathrm{GHz}$. The $x$ axis is logarithmic. 


\subsection{Microwave Phase and Spectrum Analyzer}

Traditionally, by feeding two microwave signals into a mixer circuit and detecting the voltage at the output, the phase of the microwave signal is determined. A similar principle can also be implemented in the MTJ-based microwave detectors but with a much simpler circuit. As indicated in Figure 3(a), a microwave signal under the test of $A \cos \left(\omega_{1} t+\phi_{1}\right)$ and a reference microwave signal of $B \cos \left(\omega_{2} t+\phi_{2}\right)$ are fed into two CPWs with a common short at the center.

The two CPWs are well isolated with crosstalk less than $-50 \mathrm{~dB}$. Therefore, both of the microwave signals get reflected at the common short. As a result, the microwave magnetic field generated at the common short is proportional to the linear superposition of the two microwave signals $A \cos \left(\omega_{1} t+\phi_{1}\right)+B \cos \left(\omega_{2} t+\phi_{2}\right)$. The microwave-induced voltage is proportional to the square of the total microwave magnetic field as follows:

$$
\begin{aligned}
& \Delta V \propto\left\langle A \cos \left(\omega_{1} t+\phi_{1}\right)+B \cos \left(\omega_{2} t+\phi_{2}\right)\right\rangle^{2} \\
& =\frac{A^{2}+B^{2}}{2}+A B \cos \left[\left(\omega_{1}-\omega_{2}\right) t+\left(\phi_{1}-\phi_{2}\right)\right] \\
& +A B \cos \left[\left(\omega_{1}+\omega_{2}\right) t+\left(\phi_{1}+\phi_{2}\right)\right] .
\end{aligned}
$$

Based on this equation, a microwave spectrum analyzer can be realized. If the MTJ is excited by two microwaves with different frequencies $\omega_{1}$ and $\omega_{2}$, a beat signal proportional to $A B \cos \left(\omega_{1}-\omega_{2}\right) t$ can be generated according to Equation (5). This term has a very low frequency when $\omega_{1}$ and $\omega_{2}$ are close to each other, and can be easily detected. An experiment is carried out to demonstrate such an effect, as shown in Figure 3(a). A signal under test and a reference signal at $2.000060 \mathrm{GHz}$ are fed into the structure and interfere at the MTJ. Under a constant current bias, a beat signal is generated. The signal is then filtered, amplified, and detected in the time domain with an oscilloscope. When the microwave signal under test has a nominal frequency of $2.000062 \mathrm{GHz}$, the generated beat signal has a frequency of about $2 \mathrm{kHz}$, which can pass through the filter and be detected, as shown in Figure 3(b). However, if the signal under test has a nominal frequency of $2.000000 \mathrm{GHz}$, the beat frequency would be $60 \mathrm{kHz}$, which is too high for the filter and no significant peak in the frequency domain is observed. The random signal in the time domain curve is due to the noise in the MTJ and other electronic instruments in this system. The Fourier transformation of this signal shows a sharp peak near $1750 \mathrm{~Hz}$, which is in good agreement with the nominal value. The frequency sensitivity in this experimental demonstration is on the order of $\mathrm{kHz}$, which is limited by the resolution of the band pass filter and the oscilloscope. The frequency sensitivity can be further improved with more sophisticated electronic instruments.

With two microwaves having the same frequency, Equation (5) is simplified as

$$
\Delta V=\frac{A^{2}+B^{2}}{2}+A B \cos \left[\phi_{1}-\phi_{2}\right]
$$

The phase shifter in the reference signal path determines the phase of the test signal as shown in Figure 3(c). The microwave is first split into two branches with a microwave power divider. The left branch is directly fed into the left side of the $\mathrm{CPW}$, while the right branch goes through a microwave phase shifter and fed into the right side of the CPW. The relative phase between the two microwaves can be continuously adjusted. In the experiment, the microwave power is fixed at $0 \mathrm{dBm}$ and the frequency is at $2 \mathrm{GHz}$. The voltage response has a sinusoidal dependence with the additional phase difference tuned by the phase shifter. A control measurement is also performed by placing one of the microwave probes onto an adjacent identical CPW pattern, as indicated in Figure 3(d). The experimental result shows that there is a weak phase dependence with a half periodicity due to dimensional resonance, when the microwave signal from one side of the CPW short is transmitted into the other side of the CPW. Inserting a microwave isolator in the transmission line can eliminate this multiple scattering of the microwave signal. 

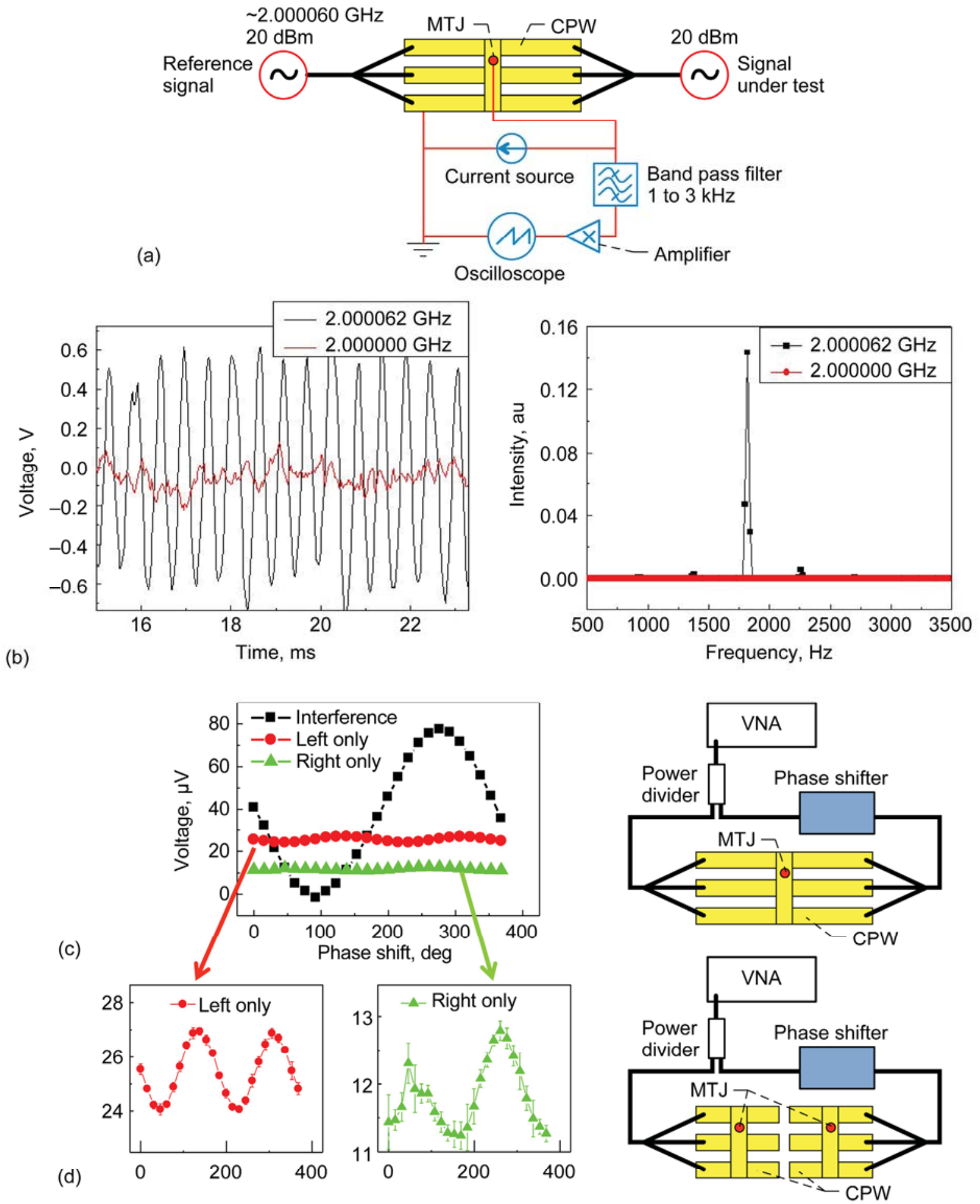

Figure 3.-(a) Experimental setup for the demonstration of the MTJ as a microwave phase and spectrum analyzer. The MTJ is biased with a constant current source, and the signal is filtered by a band pass filter and then amplified. The beat signal is detected by an oscilloscope. (b) Real time beat signals detected by the oscilloscope (left), and the Fourier transformations of the signals in frequency domain (right). (c) Schematics of the circuit for microwave phase detection. The MTJ responses to the interference of the two microwaves, corresponding to the "interference" curve. The electric detection circuit is the same as in Figure 1 and is omitted for simplicity. (d) Schematics of the circuit for control measurement. The MTJ responds to the microwave in each branch separately, corresponding to the "left only" and "right only" curves. 


\subsection{Conclusion}

In summary, we have performed a systematic study of the microwave-induced resistance change in MTJs. The quadratic dependence of resistance change with respect to microwave magnetic fields makes MTJs potential microwave detectors. The detector has its own unique features such as sensitivity to microwave magnetic fields. By integrating a mixer circuit, the microwave phase and frequency detection is demonstrated, making it possible to use an MTJ for an on-chip phase and spectrum analyzer.

\section{References}

1. E.Y. Tsymbal and I. Zutic, Handbook of Spin Transport and Magnetism. Boca Raton: Chapman and Hall/CRC, 2010.

2. D.D. Awschalom and M.E. Flatte, "Challenges for semiconductor spintronics," Nature Physics, vol. 3, no. 3, pp. 153-159, Mar 2007.

3. N. Nagaosa, "Spin currents in semiconductors, metals, and insulators," Journal of the Physical Society of Japan, vol. 77, no. 3, p. 031010, 15 Mar 2008.

4. S. Parkin, X. Jiang, C. Kaiser, A. Panchula, K. Roche, and M. Samant, "Magnetically engineered spintronic sensors and memory," (Invited Paper), Proceedings of the IEEE, vol. 91, no. 5, pp. 661680, May 2003.

5. A.A. Tulapurkar, Y. Suzuki, A. Fukushima, H. Kubota, H. Maehara, K. Tsunekawa, D.D. Djayaprawira, N. Watanabe, and S. Yuasa, "Spin-torque diode effect in magnetic tunnel junctions," Nature, vol. 438, no. 7066, pp. 339-342, 17 Nov 2005.

6. J. Zhu, J.A. Katine, G.E. Rowlands, Y.-J. Chen, Z. Duan, J.G. Alzate, P. Upadhyaya, J. Langer, P.K. Amiri, K.L. Wang, and I.N. Krivorotov, "Voltage-induced ferromagnetic resonance in magnetic tunnel junctions," Physical Review Letters, vol. 108, p. 197203, 9 May 2012.

7. A. Wirthmann, X.L. Fan, Y.S. Gui, K. Martens, G. Williams, J. Dietrich, G.E. Bridges, and C.-M. Hu, "Direct phase probing and mapping via spintronic Michelson interferometry," Physical Review Letters, vol. 105, p. 017202, 30 Jun 2010.

8. X. Fan, R. Cao, T. Moriyama, W. Wang, H.W. Zhang, and J.Q. Xiao, "Magnetic tunnel junction based microwave detector," Applied Physics Letters, vol. 95, no. 12, p. 122501, 21 Sep 2009.

9. T.L. Gilbert, "A phenomenological theory of damping in ferromagnetic materials," IEEE Transactions on Magnetics, vol. 40, no. 6, pp. 3443-3449, Nov 2004. 

